\title{
The GooD Pregnancy Network: an alternative approach for gestational diabetes.
}

\author{
Amaju Ikomi 1,*and Shaheen Mannan ${ }^{2}$ \\ 1 Basildon Hospital, Mid and South Essex NHS Foundation Trust, UK; majik@doctors.org.uk \\ 2 Basildon Hospital, Mid and South Essex NHS Foundation Trust, UK; shaheen.mannan1@nhs.net \\ * Correspondence: majik@doctors.org.uk
}

\begin{abstract}
Basildon and Thurrock University Hospital in the East of England region of the United Kingdom (U.K), witnessed rapidly increasing numbers of pregnant women with diabetes, causing overburdened specialist clinics, poorer patient experience and worsening clinical outcomes. This prompted the multidisciplinary team's remodelling of care pathways, launching the General ownership of Diabetes (GooD) Pregnancy Network in 2014. Contrary to conventional limitation of care to specialist diabetes antenatal clinics, this novel initiative highlights contemporary necessity to equip and empower all maternity stakeholders to deliver basic care of gestational diabetes (GDM). It strategically connects a Midwife Tele-Clinic "hub" to Educating Gestational diabetics Group Sessions (EGGS) and standard antenatal clinics. Patients were key partners, regularly participating in feedback surveys and promoting public awareness by co-producing local newspaper articles that served up their stories as case studies. Furthermore, the EGGS "faculty" includes a former GDM patient whose video testimony has inspired almost 2000 patients and their families; aiming to foster long term healthy lifestyle changes. Final summative evaluation in November 2019 showed the new culture of wider consciousness has shortened 'diagnosis to first consultation' intervals and eliminated overbooked specialist clinics (none since January 2016), without further worsening of clinical outcomes. It also boosted research recruitment and avoided additional running costs to the tune of $£ 66,384$ a year.
\end{abstract}

Keywords: Pregnancy; Diabetes; Screening, Lifestyle

\section{Introduction}

It is recognised that the prevalence of gestational diabetes mellitus (GDM) varies according to different populations, but the common trend across the world is rising levels of this condition. Such increases are fast becoming a major health problem [1-3] and the trend is thought to be partly explained by advancing of maternal age and increasing rates of obesity [4]. Also, prevalence rates of GDM are higher in ethnic minority populations and therefore there is a well-recognised positive association between increasing racial/ cultural diversity in Western populations and rates of GDM [5]. Basildon and Thurrock University Hospital is a University Hospital in the East of England region of the United Kingdom, with an annual delivery rate of approximately 4500 deliveries a year. Routinely collated annual service data at this hospital had demonstrated a $200 \%$ increase in the prevalence of diabetes in pregnancy between 2004 and 2013, predominantly due to gestational diabetes mellitus (GDM). Members of the specialist maternity team had increasingly recognised the adverse effect of this increased workload on care quality and in August 2013, we received our first ever written complaint relating to clinic waiting time. A significant sentinel 'event' was the increase in the number of diabetes related stillbirths, which peaked at three deaths over a four-month period (July to October 2013). The Root Cause Analysis investigation reports highlighted a recurring theme of suboptimal clinical decisions in overburdened specialist clinics. 
Once concerns were formally raised, a multidisciplinary scoping team was set up to assess the scale of the problem and examine for possible contributing factors, with a view to finding a way forward. This team consisted of a consultant obstetrician, a consultant diabetologist, a specialist diabetes midwife and a specialist diabetes nurse. For a better understanding of the scale of our caseload challenge, the team reviewed the routinely collated maternity diabetes service data for 2013 and the 2013 Public Health England Local Authority Health Profile report for our catchment population. In the UK, Public Health England was an executive agency of the Department of Health and Social Care in England tasked to protect and improve health and wellbeing and reduce health inequalities. This agency was responsible for the production of Local Authority Health Profiles reports that provide an overview of health for each local district in England. The reports contain data on a range of indicators for local populations, highlighting issues that can affect health in each locality. The profiles are intended to help local government and health services make plans to improve the health of their local population and reduce health inequalities.

The team review of our local data came up with some stark findings. The first significant revelation was that the year had marked a major inflection point in our prevalence of diabetes in pregnancy, rising from 67.3 per 1000 deliveries in 2012 to 91.6 per 1000 in 2013. The second noteworthy finding was that national census data (2001 - 2011) had demonstrated a 3-fold increase in the proportion of ethnic minority residents locally [6]. In our catchment population of Thurrock, the proportion of ethnic minority residents (Asian, African, Caribbean, Mixed and Other) had risen from 6.1\% in 2001 to $18.4 \%$ in 2011. This rate of increase was faster than the national average and the trend was predicted to continue. The trend held significance as the younger child-bearing generation was clearly more ethnically diverse compared to their parents and the data showed that many of the ethnic minority groups were displaying a higher prevalence of obesity $(21.5-29.4 \%)$ than the national average $(19.8 \%)$. The variation in obesity prevalence extended across all age groups, including children [7] (p.7).

The final concern was an ongoing rise in the overall rate of adult obesity. This trend dated back to 2006 and culminated as worse than the national average in the 2013 health profile reports for both the Thurrock and Basildon districts we serve $[8,9]$. They reported that $28.1 \%$ and $26.7 \%$ of adults in Thurrock and Basildon respectively were obese compared to the national average of $24.2 \%$. This trend remained evident and was highlighted in Thurrock Council's Joint Strategic Needs Assessment report on "Whole Systems Obesity" in 2017 [7]. It showed that between 2013 and 2015, the problem of obesity remained alarmingly high in Thurrock with $69.4 \%$ of adults registered as overweight or obese, compared to the England average of $61.3 \%$. Sadly, by 2019 , there was still no sign of abatement with the 2019 health profile reports showing $72.8 \%$ and $69 \%$ of adults in Basildon and Thurrock respectively, were overweight or obese compared to a national average of $62 \%$ [10, 11$]$.

Due to the emerging picture, further increases in the caseload of our diabetes antenatal service were strongly predicted. UK national guidelines [12] state that all diabetic patients in pregnancy should be routinely reviewed in a specialist diabetes antenatal clinic. However, due to the already overwhelming workload, not to speak of even further anticipated increases due the local demographic trends, the team realised a crucial need to adapt the service to meet the needs of the increasingly diverse community it served. We therefore set ourselves the pertinent challenge of unfunded remodelling of care pathways to safely deal with increasing caseloads. At a time of ever-widening gaps between hospital income and expenditure, we knew we faced a significant hurdle but also realised that not addressing the issue would potentially be detrimental to long term patient safety and experience, and the reputation of our maternity unit. This fuelled a decision to embrace innovation and apply quality improvement (QI) methodology in trying to find a solution. 


\section{Materials and Methods}

\subsection{Design:}

The primary objective was to reduce the rate of overbooked specialist diabetes antenatal clinics and the rate of delays in first clinic appointments following a diagnosis of GDM. The second objective was to ensure that any implemented interventions did not result in worsening of relevant clinical outcomes that were routinely collated as part of the hospital annual service data. These clinical outcomes were therefore regarded as balancing measures, whereby monitoring of these outcomes would help to ensure that our interventions did not have an unacceptable safety profile. Irrespective of achieving the primary objective, an associated negative trend in any of the balancing measures was judged to be counterproductive. It was therefore agreed from the outset that without positive or static trends in the balancing measures, the project could not be deemed a success.

Following implementation of our planned interventions, we applied plan, do, study, act (PDSA) cycles [13] as our QI methodology tool for formative evaluation and this enabled prospective adjustments during the project time frame.

The definitive final summative evaluation was done in November 2019. The setting for this evaluation was our maternity unit, with an outreach that included community midwifery clinics. The subjects were patients with a working diagnosis of diabetes in pregnancy based on the 2010 International Association of Diabetes and Pregnancy Study Groups (IADPSG)/ World Health Organisation (WHO) 2013 [14,15,] diagnostic criteria between January 2014 and December 2018. The evaluation involved annual reviews of routinely collected data and consisted of 2970 diabetic pregnancy outcomes and corresponding outpatient clinic logs.

\subsection{Measurement:}

The team gleaned baseline information by analysing routinely collated maternity diabetes service data and selected the problematic indicators in the 2013 dataset to constitute a list of chosen measures for monitoring as part of the evaluation process.

The selected process measures were the percentage of months every 6 months when specialist clinic capacity (90 clinic slots per month) was exceeded, and the rate of first clinic attendance within 7 days of GDM diagnosis. The selected clinical outcome measures (balancing measures) were: live birth rate in Type 1 and 2 diabetics, big baby (birth weight $>4 \mathrm{~kg}$ ) and caesarean section rates in GDM cases.

At the outset, the listed process measures were reviewed 3-6 monthly and for the balancing measures this was done yearly, unless earlier reviews were warranted based on safety incident reports. All results were plotted sequentially to aid visualisation of trends and reported in shared annual service reports, each detailing the range of plan, do, study, act (PDSA) activities for the year.

For the patient feedback survey, we used a locally designed questionnaire (Appendix 1) which included closed satisfaction statements and response choices were based on a five-point Likert scale, ranging from $1=$ strongly disagree to $5=$ strongly agree. This type of scale was used because it is easily understood by respondents, is useful for gauging opinions and feelings, and offers the option of negatively worded responses. The questionnaire also provided an optional section for open-ended comments. When it came to analysis of the questionnaire responses, the main focus was on statements $2-5$ which dealt with the four main educational objectives, including psychological adjustment. As this was not a validated questionnaire, a total score was not created for each respondent and the results for each question were analysed separately.

\subsection{Intervention strategy:}

This consisted of 3 work streams which ran in parallel throughout the duration of the project.

1. Remodelling and implementation of a new care pathway: 
The first step was a series of brainstorming meetings of the multidisciplinary team members and this conceived the idea of the GooD (General ownership of Diabetes) pregnancy network, an innovative initiative that emphasises contemporary necessity to equip and empower all maternity stakeholders to deliver the basic management requirements of gestational diabetes. Contrary to conventional limitation of care to specialist diabetes antenatal clinics, it advocates setting up of a service hub of Midwife led Tele-consultation clinics and strategic linkage of these to group clinics (Educating Gestational diabetics Group Sessions: EGGS) [16 -19], specialist clinics and standard antenatal clinics, thereby creating a shared care network. We then took a bottom-up approach by informally engaging with both staff (including community midwives and line managers) and patients, about this idea, towards the end of 2013. The majority were positively receptive to the proposal and these discussions informed the final decision to implement this new care framework in January 2014. After initial set up, a pilot questionnaire based feedback from 17 service users (January - March 2014) showed 100\% preferred the option of tele-consultation to physical attendance. An overriding theme of greater patient convenience based on time off work, childcare arrangements and parking costs became evident. From March 2014 onwards, we proceeded to embed the new pathway and outlined a five-year timeframe during which we aimed to hopefully demonstrate longer term benefits and sustainability of the new initiative.

2. Staff engagement and behavioural change:

Initial consultations with general staff members of the maternity workforce highlighted a need to widen consciousness and clinical knowledge of GDM to enable a positive impact of the new shared care pathway. This proved to be our biggest challenge, especially as management had declined our request to incorporate this domain of care into the mandatory training programme. We coped by grasping 'windows of educational opportunity 'such as rotational attendance of specialist clinics (including EGGS) by non-specialist staff, remote support for non-specialist staff by the specialist team, selecting and training diabetes champions in all clinical areas, holding weekly multidisciplinary team meetings with an open invitation to non-specialist staff members, launching a departmental poster campaign reminding all staff to routinely enquire about glycaemic control when in contact with diabetic women and to escalate when necessary, general circulation of diabetes annual reports, development and implementation of home grown decision support aids. The DRRiP (Diabetes Related Risk in Pregnancy) score is our simple checklist tool that converts evaluation of discrete clinical parameters into a single composite score, thus creating a common language between patients, midwives and doctors. DIALD (Diabetes And Latest Delivery date) is our decision aid that stipulates the timing of delivery according to the emerging clinical picture.

3. Collaboration with patients

Public health data shows that after delivery, women from Basildon and Thurrock face a greater risk of cardio metabolic complications and lower life expectancy. For this reason, we decided to make public engagement a crucial component of this initiative and made a commitment to Patients and Public Involvement and Engagement (PPIE) [20]. After the pilot survey confirmed collective endorsement of the new tele-consultation service, all GDM patients were routinely offered participation in feedback surveys, revealing the consequential extent of empowerment and psychological adjustment following the group sessions. Furthermore, patients joined to co-produce a series of 5 newspaper articles since 2015, serving up their stories as case studies promoting 'general ownership'. Meaningful public engagement was also evident in 10,882 views of our 2016 social media post and as a direct consequence, the EGGS group clinic 'faculty' has since included a former GDM patient. Her involvement has enabled the group clinics to always end on an encouraging note by showing a short film of her testimony. Despite requiring insulin treatment 16 years earlier, she remained diabetes free and both her teenage children were lean and fit, 
reiterating tangible linkage of parental lifestyle choices to childhood obesity and glycaemic impairment [21 - 23].

\section{Results}

\subsection{Process measures}

3.1.1. Specialist Clinic capacity:

Baseline data from outpatient clinic logs showed overbooked specialist (diabetes) antenatal clinics with more than 90 face to face consultations occurred every month $(100 \%)$ from July to December 2013. After implementation of the intervention strategy, the rate of months when specialist clinic capacity was exceeded reduced to $8 / 12(66.6 \%)$ in 2014 with further reduction to 5/12 (41.6\%) in 2015 and has remained at $0 \%$ since 2016. (Figure $1)$.

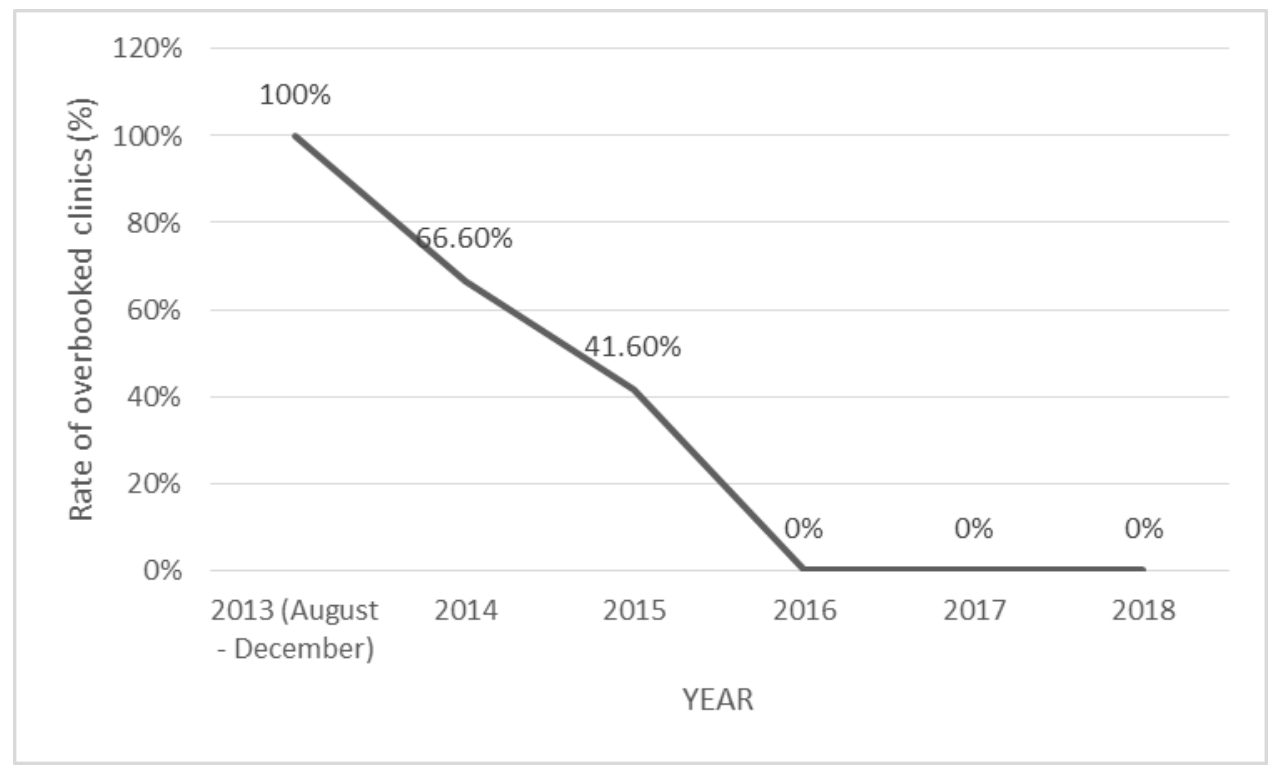

Figure 1. Trend in overbooked specialist diabetes antenatal clinic rates following implementation of the GooD Pregnancy Network in January 2014.

3.1.2. Rate of first clinic attendance within 7 days of GDM diagnosis:

In February 2015, new national guidelines stipulated a new standard in ensuring first clinic attendance within 7 days of a new diagnosis of GDM [12]. For this reason, this process measure was introduced as part of the monitoring programme. During the year we noticed a gradual reduction in performance, hitting a nadir of $31.8 \%$ at the start of 2016. This speeded up our planned introduction of group clinics which were implemented in March 2016, immediately quadrupling our weekly capacity for seeing new patients. Significant improvement was apparent by the end of the $2^{\text {nd }}$ PDSA cycle in May 2016 but this was short-lived with a subsequent nadir of $28.75 \%$ at the end of the $4^{\text {th }}$ PDSA cycle in November 2016. It transpired that this was a direct consequence of relocation of the pathology reporting system to an off-site facility, resulting in delays in reporting of Oral Glucose Tolerance Test results. Following a resolution meeting with the relevant biochemistry team, the issues were addressed and the frequency of PDSA cycles was increased to monthly. Consequently, attendance rate within 7 days of GDM diagnosis soared to consistently hover around $94 \%$ between February and July 2017. The details are shown in Figure 2. 


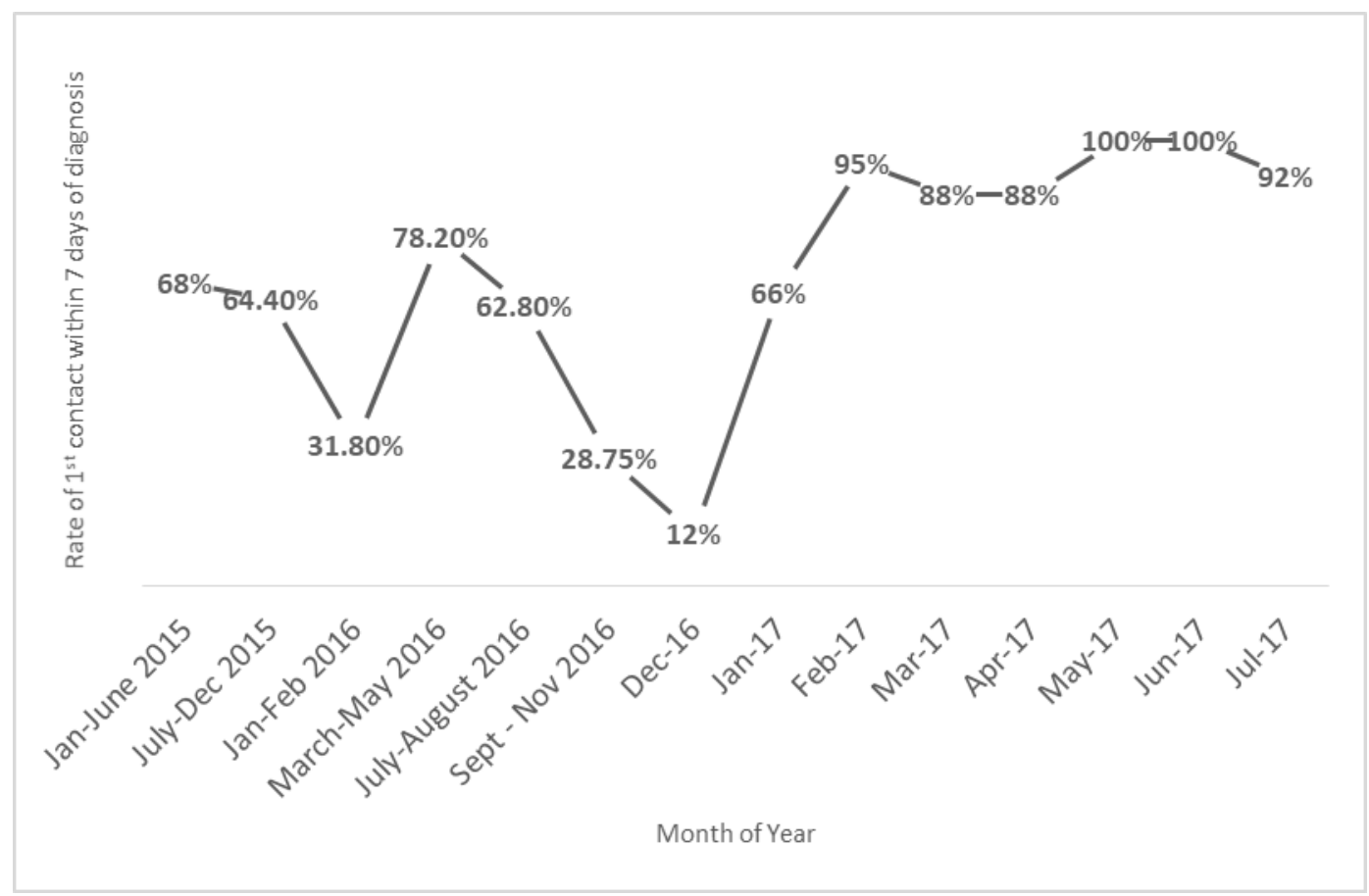

Figure 2. Trend in rate of first clinic appointment within 7 days of a new diagnosis of GDM, before and following implementation of group clinics in March 2016.

\subsection{Balancing clinical outcome measures}

\subsubsection{Live-birth rates in Type 1 and 2 diabetic women:}

Baseline annual outcome data unveiled our lowest ever, live birth rate (92.6\%) in Type 1 and 2 diabetics (highest risk patients); significantly worse than the corresponding national rate (98.6\% in 2013) according to the National Pregnancy in Diabetes (NPID) Audit report 2013 [24]. After implementation of the intervention strategy, the rate of live-births of Type 1 and 2 diabetic women increased to 'bridge the gap' in 2014 (97.3\% vs. national rate of $98.9 \%)$, and finally 'hit the mark' in 2015 (100\%) where it has remained since. (Figure 3). Concurrently in this group of patients, there was continuous improvement in our rate of ideal 3rd trimester glycaemic control (HbA1c level of $<48 \mathrm{mmol} / \mathrm{mol}$ ), achieving our best ever result of $72.7 \%$ in 2018 , compared to the corresponding national rate of $57.5 \%$. 


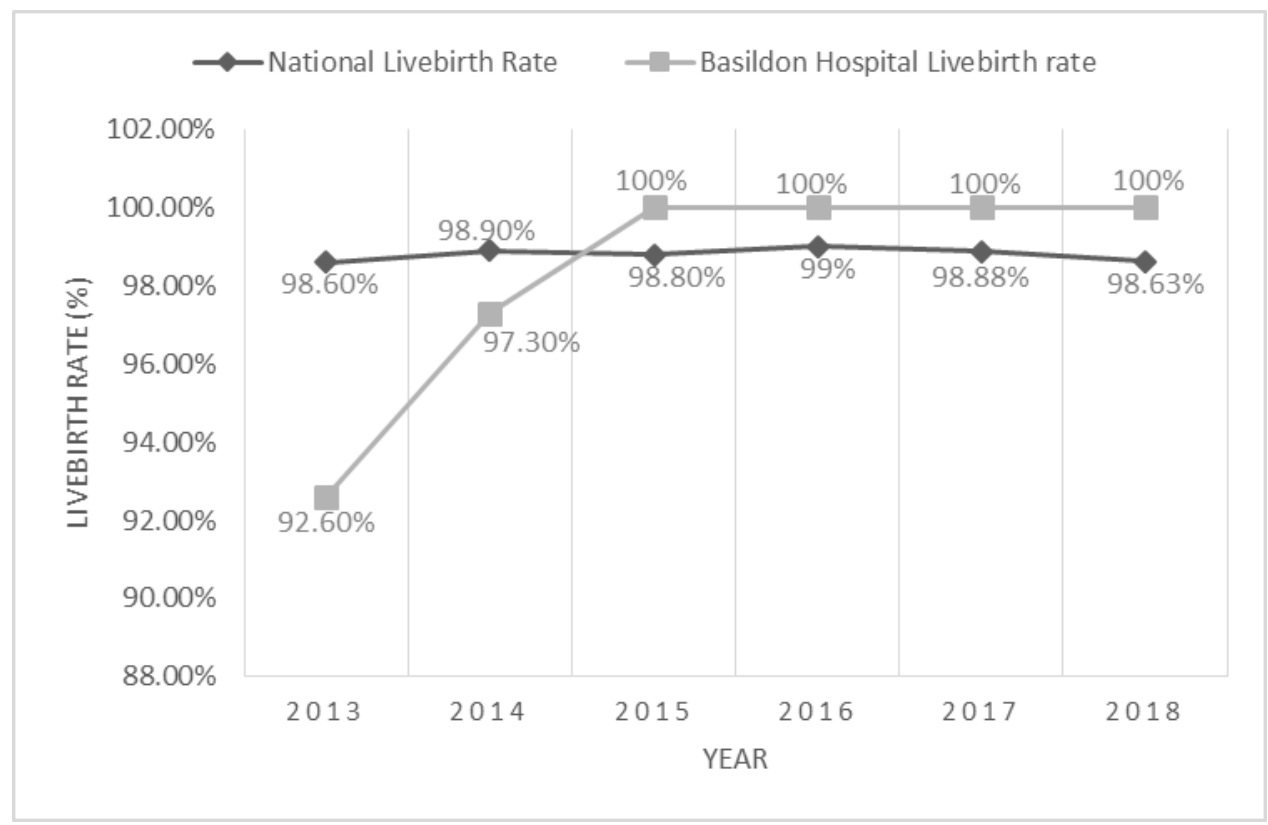

Figure 3. Trends in live birth rates in Type 1 and 2 diabetic women following implementation of the GooD Pregnancy Network in January 2014.

3.2.2. Caesarean section and big baby rates in GDM women:

Baseline data showed suboptimal rates of some relevant outcomes like big baby $(>4 \mathrm{~kg})$ which occurred in $13.2 \%$ of our GDM cases and obstetric interventions like Caesarean section which occurred in $43 \%$ of our GDM cases. After implementation of the intervention strategy, we witnessed a downward trend in annual caesarean section and big baby rates compared to the 2013 baseline, averaging 36.9\% and 7.7\% respectively between 2014 and 2018. (Figure 4). The levelling up of the downward trend of both rates noticeably seemed to be related to a reduction in the rate of pharmacological treatment after 2016. Subsequently, team reflection about this finding has resulted in a strategic lowering of our threshold to commence pharmacological treatment with Metformin and/ or Insulin.

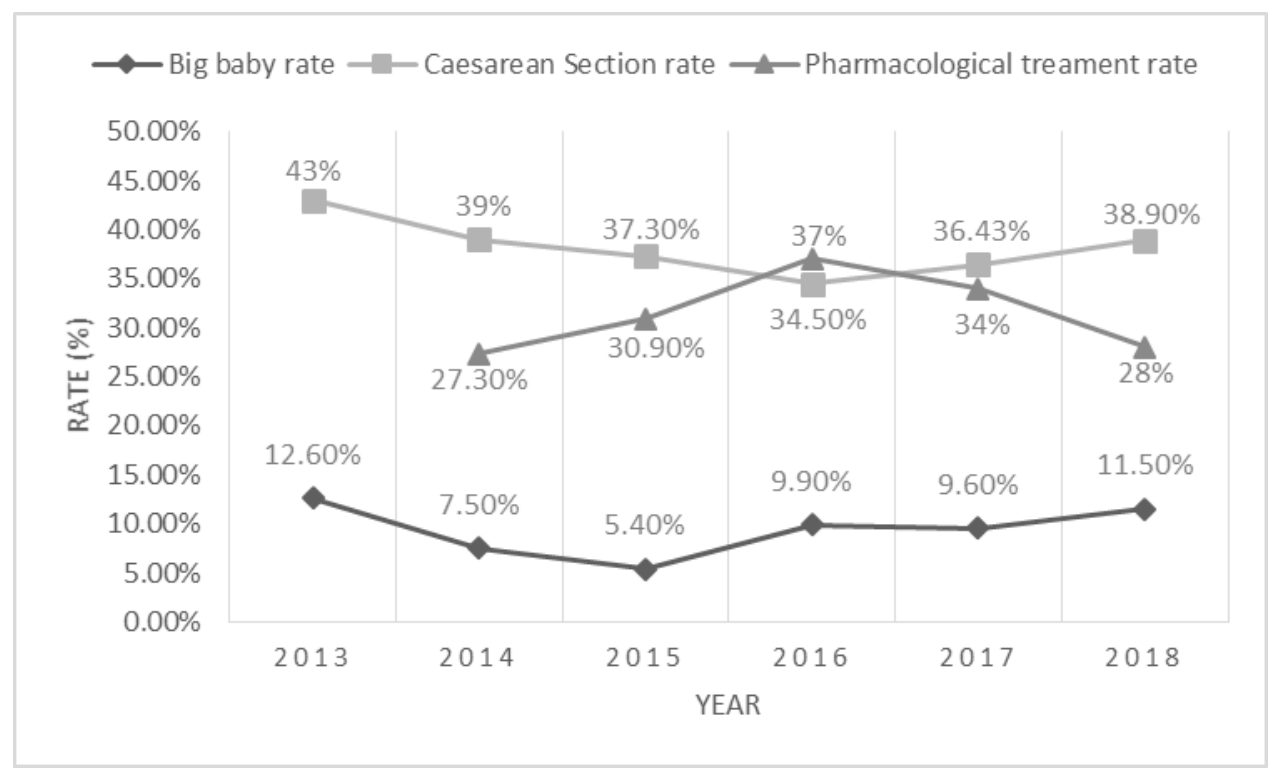

Figure 4. Trends in big baby, caesarean section and pharmacological treatment rates in gestational diabetic women following implementation of the GooD Pregnancy Network in 2014. 


\subsubsection{Patient satisfaction:}

A 2017 survey of 338 GDM patients showed roughly $98 \%$ of respondents concurred on successful achievement of the four main educational objectives, including psychological adjustment. (Table 1). The central theme was positive engagement due to support received from carers. However, patients also expressed concerns about 'general ownership' potentially detracting from the attentiveness of the specialist team. The 2019 survey of 231 patients reaffirmed the previous result (Table 2) but also revealed that $96 \%$ valued diabetes support from their routine carers.

Table 1. Patient survey feedback following attendance of group clinics: distribution of Likert scale responses by 338 respondents in 2017.

Closed statement
Strongly agree or agree

\section{Neither agree nor disagree}

Disagree or strongly disagree
332/ 338 (98.2\%)

6/ $338(1.8 \%)$

0/ $338(0 \%)$
$6 / 338(1.8 \%)$

4/ $338(1.2 \%)$

2/ $338(0.6 \%)$

The session met my expectations.

$329 / 338(97.3 \%)$ $332 / 338(98.2 \%)$

(4)

(2)
I feel confident about making positive changes for gestational diabetes.

(n)

$333 / 338(98.5 \%) \quad 5 / 338(1.5 \%) \quad 0 / 338(0 \%)$

(n)

Table 2. Patient survey feedback following attendance of group clinics: distribution of Likert scale responses by 231 respondents in 2019.

\section{Closed statement}
Neither agree nor disagree

Disagree or strongly disagree 


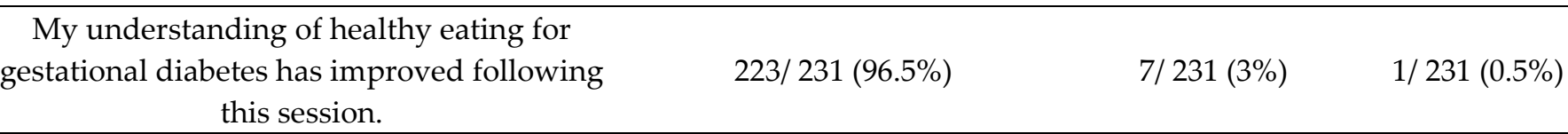

I understand how to use a blood glucose monitor after this session. $231 / 231(100 \%)$ $0 / 231(0 \%)$ $0 / 231(0 \%)$

The session met my expectations.

$226 / 231(97.8 \%) \quad 5 / 231(2.2 \%) \quad 0 / 231(0 \%)$

I feel confident about making positive changes for gestational diabetes. $225 / 231(97.4 \%)$ $6 / 231(2.6 \%)$ $0 / 231(0 \%)$

3.2.4. Additional observations:

The camaraderie of the group clinic also boosted recruitment to national research studies e.g. for the Diabetes Alliance for Research in England (DARE) study [25], this clinic enrolled $640(38 \%)$ of the hospital's 1677 recruits, resulting as the top recruiter in North Thames region and fifth nationally.

There were also cost savings due to the newly implemented care pathway. Prior to the changes, all antenatal consultations were done by members of the specialist multidisciplinary diabetes team (obstetrician, diabetologist, specialist midwife, specialist nurse and nutritionist) in specialist (diabetes) antenatal clinics. These were constantly overbooked and conventionally the answer to this problem would have been to increase the number of these specialist clinics to deal with the ever increasing caseload. However, our solution of simply converting our weekly conventional 'first contact' specialist (diabetes) clinic to a group clinic resulted in a four-fold increase in clinical capacity, without any increase in the salary costs of the care providers (specialist midwife and nutritionist). By preventing the need for three additional conventional 'first contact' clinics a week and taking account of the salary costs of relevant specialist care providers at the time of implementation, we calculated savings to the tune of $£ 1728$ a month.

The second aspect of cost prevention related to conducting remote tele-consultations instead of our previous practice of face to face $1^{\text {st }}$ follow-up consultations in the weekly multidisciplinary specialist clinic. On average 91 tele-consultations were performed each month, with a phone cost of $£ 259$ (assuming a phone call tariff of $19 \mathrm{p} /$ minute (landline to mobile) and fifteen-minute consultations) and midwifery time cost of $£ 450$, all leading to an estimated monthly running cost of $£ 709$. An equivalent number of face to face conventional consultations with the whole multidisciplinary team would have involved running eight additional multidisciplinary specialist clinics each month at a monthly salary cost of $£ 4513.8$. This amounts to cost savings of $£ 3804.8$ each month.

We retained our previous practice of scheduling all $2^{\text {nd }}$ follow-up appointments for the specialist clinic and this was cost neutral as this clinic was previously established and running. However, arrangements for final follow-up appointments were changed to being performed by non-specialists in standard antenatal clinics. By encouraging the use of a range of decision aids, we were able to ensure consistency in the timing of delivery by non-specialist clinicians. This final aspect of the new care pathway was also cost neutral as the standard antenatal clinics were already established and running before the intervention strategy. In total, the costing savings were therefore estimated at $£ 66,384$ a year. 
We realise this number may not reflect an actual financial saving as we did not reduce spending in real terms. However, the fact that improvements occurred without the usual need to increase spending represents a cost effective approach. The cost analysis is summarised in Table 3.

Table 3. Cost implications of implementing the new GooD Pregnancy Network pathway.

\section{Type of appointment}

$1^{\text {st }}$ contact

$1^{\text {st }}$ follow-up
Group clinic

Type of antenatal clinic

Prevented the need

for 3 additional

conventional $1^{\text {st }}$

contact clinics a

week.
Cost savings of

$£ 1728$ /month

Cost status

Prevented the need

for 8 additional

multidisciplinary Cost savings of

specialist clinics/

$£ 3804.8$ / month.

Tele - consultation clinic month.

$2^{\text {nd }}$ follow-up

Specialist clinic

Was already run-

Cost neutral. ning.

Final follow-up

Standard clinic

(n)


tals. This led to an increased number of non - English speaking patients requiring diabetes education with the support of language interpreters, resulting in longer and more frequent conventional face to face consultations. Our records show the language interpreter requiring patients originated from 14 different countries worldwide, ranging from Brazil in the west to Vietnam in the east. All these factors and the well reported higher rates of poorer glycaemic control associated with the COVID-19 era fuelled a recognised need for more clinician time.

Fortunately, the solution lay in the fact that our network had established a tele-consultation service as far back as 2014. At that time we had no idea that virtual consultations would become the norm during the pandemic crisis and when it happened we found ourselves well placed to adapt quickly. At the height of the pandemic in July 2020, we simply converted the tele-consultation service to a digital platform using wireless blood glucose meters and the GDm-Health app [26]. This has allowed for near real-time remote monitoring of glycaemic control in most of our patients and shortened the average time of our scheduled virtual consultations. Our records showed a $44.5 \%$ reduction in virtual consultation time between January and March 2021, amounting to 85 hours of released clinician time. This unblocked more time for the team to focus on the additional clinical challenges that arose due to the Covid - 19 pandemic.

Another consequence of cessation of group gatherings due to the Covid - 19 pandemic was the temporary suspension of diagnostic oral glucose tolerance tests. This led the Royal College of Obstetricians and Gynaecologists (RCOG) to issue new national guidance on the diagnosis of gestational diabetes mellitus (GDM). It recommended avoiding the 'gold standard' 2-h oral glucose tolerance test (OGTT), instead stipulating random glucose and $\mathrm{HbA}_{1 c}$ tests at booking, followed by $\mathrm{HbA}_{1 c}$ and fasting glucose testing (or random glucose if a fasting sample is not feasible) at $24-28$ weeks gestation [27]. The associated likelihood of under-diagnosis (false-negatives) due to incomplete OGTTs, raised our concerns about a considerable number of women potentially being left untreated.

This prompted us to apply Bayesian modelling in generating an estimate of the number of women facing this risk. We reanalysed our previously published OGTT data [28] and this revealed a GDM pre-test probability of 0.182 and a negative likelihood ratio of 0.362 for an isolated normal fasting glucose result $<5.1 \mathrm{mmol} / \mathrm{l}$ (WHO 2013 diagnostic threshold). These calculations were applied to the 3 steps of Bayes formula and projected a failed detection rate of no greater than $7.4 \%$, if the new guidance were to be adopted [29]. Under the circumstances we judged this to be an acceptable level of under-diagnosis and therefore chose to implement the new guidelines in May 2020, also encouraging other maternity units to do the same. One year later (June 2021), we retrospectively analysed routinely collected data in our maternity database, aiming to compare GDM case numbers before and after implementation of the new RCOG guidelines. The 'expected' number of COVID era cases was modelled on historical average yearly increases in GDM numbers. We found a close similarity between our estimated numbers of expected and actual "missed" cases (46 vs. 45), verifying strong predictive accuracy of the Bayesian modelling exercise and thus upholding the suitability of the RCOG COVID - 19 diagnostic pathway for our local population [30].

Our experience also highlights the advantage of utilising local datasets and underlines a role for this modelling approach in meeting the evolving needs of patients at times of crisis. Furthermore, we also believe the culture of wider consciousness embedded by our GooD pregnancy network structure helped us to maximise the detection of GDM during the pandemic.

\section{Discussion}

U.K. national guidelines [12] stress the importance of confining care of diabetes in pregnancy to specialist multidisciplinary antenatal clinics so the concept of 'general ownership' of diabetes in pregnancy is somewhat new. Following implementation using QI methodology we achieved our primary objective (improved specialist clinic capacity and 
timely first contact for GDM patients), without any worsening of the balancing clinical outcome measures.

The strengths of this project are the reporting of patient satisfaction data, application of PDSA cycles and an overall time frame that provides evidence of sustainability. The main limitation is the lack of data on other neonatal morbidity outcomes such as neonatal hypoglycaemia and admissions to neonatal intensive care units. We also recognise the limitation of not applying any statistical tests to explore the significance of the positive trend in the clinical outcomes. We deliberately omitted this step because our project was a simple before-and-after evaluation and we were highly cognisant of the inherent bias of such evaluations in relation to clinical outcomes, in the absence of an unexposed control group. For this reason, the clinical outcomes remained relegated to the status of balancing measures. That said, the noticeable positive trends were an interesting observation that now creates a fresh hypothesis that these trends may be a direct consequence of the interventions. We are of the opinion that this hypothesis should now be explored in larger prospective multi-centre controlled trials.

Staff engagement and education proved to be our biggest challenge during this operational journey. Sadly, at the outset, the management team declined our request to incorporate this domain of care into the mandatory training programme. In hindsight we now feel we should have worked harder at trying to convince them otherwise, as it became clear that instituting mandatory training for non-specialist clinicians before commencement would have enabled a smoother transition.

One of the criticisms of IADPSG/ WHO 2013 diagnostic criteria for GDM is the association with increased caseloads. However, the proven connection of this criteria to long-term risks of maternal and childhood glycaemic impairment [31, 32] makes it a crucial component of contemporary public health initiatives for the ethnically diverse population we serve. It is especially noteworthy that during the corresponding time period (2014 - 2018) there was a $44 \%$ surge in the caseload of patients with diabetes in pregnancy and our official district health profile reports showed concurrent increases in rates of overweight adults and obese children, peaking to become the highest rates in East of England region and $10^{\text {th }}$ highest of 325 national districts. This poor ranking on the health profile scale adds far-reaching value to our project, as a long-term prevention initiative. Therefore, the GooD Pregnancy Network initiative offers a pragmatic balance of immediate minimal additional resource costs against the potentially markedly reduced costs of improved perinatal outcomes and long-term health. This organisational journey demonstrates cost-effective realignment of clinical capacity to increasing demand by minimising duplication of care and improving patient empowerment. Furthermore, aside from upholding stipulated values of inter-professional education, the group clinic provides an additional dimension by enabling patients, partners and professions to learn with, from and about each other [33].

Although our maternity unit is based in the UK, we have applied WHO 2013 diagnostic criteria for gestational diabetes since 2013 and serve a highly diverse multi-ethnic pregnant population. We therefore believe the applicability of this initiative extends well beyond the borders of the UK, and so we strongly encourage all clinicians to consider which components of the range of interventions we used would best suit the populations they serve. We also recommend linkage to publicly available information resources about the health and demographics of the local population, as we did, before proceeding with an intervention strategy.

On reviewing the contemporary literature we found only a few published studies of antenatal group clinics for gestational diabetes [34, 35]. However, a common thread was that they all reported improvements in patient satisfaction, knowledge, empowerment and other outcomes. A similar tendency to improvement is also evident when examining the literature on telemedicine usage for gestational diabetes [26, 36]. Our experience therefore adds to this growing body of evidence but offers an extra dimension by demonstrating the potential benefits of applying a composite of different elements, including active patient collaboration, to generate the kind of fundamental cultural shift 
that improved operational performance and has kept our service afloat during the Covid - 19 pandemic. To the best of our knowledge, this report of the sustainability of a composite approach for gestational diabetes is the first of its kind in the medical literature. In conclusion, our described inclusive network structure is an alternative care framework that offers a 'right care, right place, right time' model for delivering the stipulated elements of care. It features a modified role for the specialist team that concentrates on the highest risk cases, whilst attentively overseeing the care of all others by their usual carers. Essentially, systems leadership has successfully eliminated elitism by demystifying definitive care, embedding a culture of wider consciousness and connectivity that enables consistency during holistic care.

The final message is simple: 'The prevalence of diabetes is set to skyrocket. Rising to this challenge requires basic involvement of all health professionals in prioritising early prevention strategies that target the parents of unborn children, embodying larger societal needs'.

Funding: This project received no external funding.

Institutional Review Board Statement: Ethical review and approval were not necessary as this was not applicable to this Quality Improvement (QI) project.

Informed Consent Statement: Not applicable due to QI project.

Data Availability Statement: QI project, not research.

Acknowledgments: Authors would like to acknowledge Sherri Smith, Janice Robbins, Kerry Stone, Una Kavanagh, Rehman Khan, Godwin Simon, Donna Southam, Joanne Hoare and Suvaran Kiss for assistance with data collection, analysis and presentation at various stages of this project.

Conflicts of Interest: The authors declare no conflict of interest.

\section{References}

1. Whicher, C.; O'Neill, S.; Holt, R. Diabetes in the UK: 2019. Diabetic Medicine 2020, 37, 242-247.

2. Harding, J.; Pavkov, M.; Magliano, D.; Shaw, J.; Gregg, E. Global trends in diabetes complications: a review of current evidence. Diabetologia 2018, 62, 3-16.

3. Khan, M.; Hashim, M.; King, J.; Govender, R.; Mustafa, H.; Al Kaabi, J. Epidemiology of Type 2 Diabetes - Global Burden of Disease and Forecasted Trends. Journal of Epidemiology and Global Health 2019, 10, 107.

4. Alwash, S.M.; McIntyre, H.D.; Mamun, A. The association of general obesity and visceral body fat with the risk of gestational diabetes mellitus: Evidence from a systematic review and meta-analysis. Obesity Research \& Clinical Practice 2021, 15(5), $425-430$.

5. Powe, C.E.; Carter, E.B. Racial and Ethnic Differences in Gestational Diabetes: Time to get serious. JAMA 2021, 326(7), 616 617.

6. Thurrock Joint Strategic Needs Assessment. Demograhics and Population Change; 1st ed.; Thurrock Council: UK, 2015; p. 15. Available at https://www.thurrock.gov.uk/sites/default/files/assets/documents/jsna-demographics-population-v02.pdf (accessed on 30th December 2021).

7. Thurrock Joint Strategic Needs Assessment. Whole Systems Obesity; 1st ed.; Thurrock Council: Thurrock, UK, 2017; p. 7. Available online: https://www.thurrock.gov.uk/sites/default/files/assets/documents/jsna-obesity-201709-v01.pdf (accessed on 30th December 2021).

8. Public Health England. Thurrock Health Profile 2013, 1st ed.; Public Health England: London, U.K., 2013; p 4. Available online: https://fingertips.phe.org.uk/profile/health-profiles/data\#page/13/ati/202/are/E06000034 (accessed on 30th December 2021).

9. Public Health England. Basildon Health Profile 2013, 1st ed.; Public Health England: London, U.K., 2013; p 4. Available online: https://fingertips.phe.org.uk/profile/health-profiles/data\#page/13/ati/201/are/E07000066 (accessed on 30th December 2021).

10. Public Health England. Basildon Local Authority Health Profile 2019, 1st ed.; Public Health England: London, U.K., 2019; p 4. Available online: https://fingertips.phe.org.uk/profile/health-profiles/data\#page/13/ati/201/are/E07000066 (accessed on 30th December 2021). 
11. Public Health England. Thurrock Local Authority Health Profile 2019, 1st ed.; Public Health England: London, U.K., $2019 ;$ p 4. Available online: https://fingertips.phe.org.uk/profile/health-profiles/data\#page/13/ati/202/are/E06000034 (accessed on 30th December 2021).

12. National Institute for Health and Care Excellence (NICE). Diabetes in pregnancy: management from preconception to the postnatal period; NICE: Manchester, U.K., 2015. Clinical guideline NG3. Available at: $\underline{\text { https://www.nice.org.uk/guidance/ng3 }}$ (accessed on 30th December 2021).

13. NHS England and NHS Improvement. Plan, Do, Study, Act (PDSA) cycles and the model for improvement. NHS England: U.K., 2021. Available at: https://www.england.nhs.uk/wp-content/uploads/2021/03/qsir-plan-do-study-act.pdf (accessed on 30th December 2021)

14. Metzger, B. International Association of Diabetes and Pregnancy Study Groups Recommendations on the Diagnosis and Classification of Hyperglycemia in Pregnancy. Diabetes Care 2010, 33, 676-682.

15. World Health Organisation (WHO). Diagnostic Criteria and Classification of Hyperglycaemia First Detected in Pregnancy. Available online: https://www.who.int/publications-detail-redirect/WHO-NMH-MND-13.2 (accessed on 30th December 2021)

16. Caballero, C. Shared medical appointments. The Nurse Practitioner 2015, 40, 1-6.

17. Guirguis, A.; Lugovich, J.; Jay, J.; Sanders, K.; Cioffi, S.; Jeffery, S.; Kravetz, J. Improving Diabetes Control Using Shared Medical Appointments. The American Journal of Medicine 2013, 126, 1043-1044.

18. Jhagroo, R.; Nakada, S.; Penniston, K. Shared Medical Appointments for Patients with Kidney Stones New to Medical Management Decrease Appointment Wait Time and Increase Patient Knowledge. Journal of Urology 2013, 190, 1778-1784.

19. Lacagnina, S.; Tips, J.; Pauly, K.; Cara, K.; Karlsen, M. Lifestyle Medicine Shared Medical Appointments. American Journal of Lifestyle Medicine 2020, 15, 23-27.

20. Hickey, G.; Chambers, M. Patient and public involvement and engagement: Mind the gap. Health Expectations 2019, 22, 607-608.

21. Zhu, Y.; Zhang, C. Prevalence of Gestational Diabetes and Risk of Progression to Type 2 Diabetes: a Global Perspective. Current Diabetes Reports 2016, 16.

22. Noctor, E. Type 2 diabetes after gestational diabetes: The influence of changing diagnostic criteria. World Journal of Diabetes 2015, 6, 234.

23. Feig, D.; Zinman, B.; Wang, X.; Hux, J. Risk of development of diabetes mellitus after diagnosis of gestational diabetes. Canadian Medical Association Journal 2008, 179, 229-234.

24. NHS Digital. National Pregnancy in Diabetes Audit - 2013. Available on line: https://digital.nhs.uk/data-and-information/publications/statistical/national-pregnancy-in-diabetes-audit/national-pregna ncy-in-diabetes-audit-2013\#: :text=Publication\%2C\%20Part\%20of,31\%20Dec\%202013 (accessed on 30 ${ }^{\text {th }}$ Oct, 2021).

25. Diabetes Alliance for Research in England (DARE) // Diabetes Genes https://www.diabetesgenes.org/current-research/dare/ (accessed Oct 30, 2021).

26. Mackillop, L.; Hirst, J.E.; Bartlett, K.J.; Birks, J.S.; Clifton, L.; Farmer, A.J.; Gibson, O.; Kenworthy, Y.; Levy, J.C.; Loerup, L.; Rivero-Arias, O.; Ming, W.; Velardo, C.; Tarassenko, L. Comparing the Efficacy of a Mobile Phone-Based Blood Glucose Management System in Women with Gestational Diabetes: Randomized Controlled Trial. JMIR Mhealth Uhealth 2018, 6(3): e71.

27. Royal College of Obstetricians and Gynaecologists (RCOG). Guidance for maternal medicine services in the evolving coronavirus (COVID - 19) pandemic. Information for healthcare professionals Version 2.1. Published Friday 24 April 2020. https://www.rcog.org.uk/globalassets/documents/guidelines/2020-07-10-guidance-for-maternal-medicine.pdf (accessed on 30th December 2021)

28. Ikomi, A.; Mannan, S.; Anthony, R.; Kiss, S. Likelihood of 'falling through the net' relates to contemporary prevalence of gestational diabetes. Diabetologia 2015, 58, 2671 - 2672.

29. Ikomi, A.; Mannan, S.; Simon, G.; Khan, R.; Smith, S.; Robbins, J.; Kavanagh, U.; Crone, D. Diagnosis of gestational diabetes during the pandemic: what is the risk of falling through the net? Diabet. Med. 2020, 37, 1782 - 1784. 
30. Mannan, S.; Ikomi, A. Accuracy of Bayesian modelling in predicting COVID - 19's impact on gestational diabetes rates. The Physician: Journal of International Health, Proceedings of BAPIO25 Silver Jubilee Conference 2021, Birmingham, U.K., $22-24^{\text {th }}$ October 2021; Bisht, S., Nageswaran, P., Gajanan, K., Daga, S., Chakravorty, I, Eds. Physicianjnl.net: U.K., Year 2021; PP-23, p 35.

31. Lowe, W.L.; Scholtens, D.M; Lowe, L.P; et al. Association of Gestational Diabetes With Maternal Disorders of Glucose Metabolism and Childhood Adiposity. Journal of the American Medical Association 2018; 320(10), 1005-1016.

32. Lowe, W.L.; Scholtens, D.M.; Kuang, A.; et al. Hyperglycemia and Adverse Pregnancy Outcome Follow-up Study (HAPO FUS): Maternal Gestational Diabetes Mellitus and Childhood Glucose Metabolism. Diabetes Care 2019, 42 (3), $372-380$.

33. Lincoln, M.; McAllister, L. Peer learning in clinical education. Medical Teacher 1993, 15, 17-26.

34. Nasso, J.; McCloskey, C.; Nordquist, S.; Franzese, C.; Queenan, R.A. The Gestational Diabetes Group Program. The Journal of Perinatal Education 2018, 27(2), 86 - 97.

35. Schellinger, M.; Abernathy, M.; Foxlow, L.; Carter, A.; Bastawros, D.; Hass, D. Improved outcomes for Hispanic patients with gestational diabetes using the Centering Pregnancy group prenatal care model. American Journal of Obstetrics and Gynaecology 2013, 208(1), S - 128.

36. Tian, Y.; Zhang, S.; Huang, F.; Ma, L. Comparing the Efficacies of Telemedicine and Standard Prenatal Care on Blood Glucose Control in Women With Gestational Diabetes Mellitus: Randomized Conrolled Trial. JMIR Mhealth Uhealth 2021, 9(5): e22881. 